\title{
Respiratorische Faktoren in der Klinischen Psychophysiologie und dem Biofeedback ${ }^{1}$
}

\author{
F.H. Wilhelma, ${ }^{a}$ R. Gevirtz ${ }^{c} \quad$ W.T. Roth ${ }^{a}, b$ \\ ${ }^{a}$ Department of Psychiatry and Behavioral Sciences, Stanford University, Stanford, CA, bDepartment of Veterans Affairs Health Care \\ System, Palo Alto, CA, 'California School of Professional Psychology - San Diego, CA, USA
}

\section{Schlüsselwörter}

Atmung · Psychophysiologie $\cdot \mathrm{pCO}_{2} \cdot$ Angststörungen · Funktionelle Herzbeschwerden · Chronische Schmerzen

\section{Zusammenfassung}

Die Atmung ist ein komplexes physiologisches System, welches viele körperliche Prozesse beeinflusst und als kritisches Bindeglied zwischen Leib und Seele fungieren kann. In diesem Artikel diskutieren wir die Physiologie und Psychophysiologie der Atmung, insofern dies für ein Verständnis einer Reihe von klinischen Syndromen, die herkömmlicherweise nicht direkt mit Atemstörungen in Verbindung gebracht werden, relevant ist. Wir stellen Befunde aus drei Bereichen vor: Panikstörung, funktionelle Herzbeschwerden und chronischer Schmerz. Die Befunde unterstützen die Annahme, dass bei einer Anzahl von körperlichen Symptomen respiratorische Faktoren entweder als Marker, als vermittelnde oder als ursächliche Mechanismen bedeutsam sind. Die mithilfe neuer Technologien mögliche ambulatorische Messung des $\mathrm{CO}_{2}$-Partialdruck $\left(\mathrm{pCO}_{2}\right)$ und der Atemkurve eröffnet neue Wege der Erforschung und Behandlung dieser Störungen. Zunächst werden Ergebnisse unserer Laborstudien dargestellt, die zeigen, dass bei Panikpatienten, im Gegensatz zu anderen Angststörungen oder normalen Kontrollgruppen, subtile, aber konsistente Atmungsanomalien vorliegen. Entgegen früheren Modellen, die die akute $\mathrm{Hy}$ perventilation als zentralen Faktor bei Angststörungen herausstellten, fokussiert diese Forschung auf respiratorischer Instabilität sowie Atemverhalten wie Seufzer (engl. sighs) als Hinweise auf eine dysfunktionale Regulierung der Atmung. Schließlich diskutieren wir verschiedene Therapieansätze, die auf dem vorgeschlagenen Mediations-Modell basieren. Angewandte psychophysiologische Methoden werden vorgestellt, die zu einer effektiven Behandlung der oben genannten Störungsbilder beitragen können.

${ }^{1}$ Deutsche Übersetzung: Alexandra Nanke und Hans-Jürgen Korn, Klinik Roseneck, Prien am Chiemsee

\section{Key Words}

Respiration - Psychophysiology $\cdot \mathrm{pCO}_{2}$. Anxiety disorders · Functional cardiac disorders - Chronic pain

\section{Summary}

Respiratory Factors in Clinical Psychophysiology and Biofeedback

Respiration is a complex physiological system affecting a variety of physical processes that can act as a critical link between mind and body. In this paper, we discuss the physiology and psychophysiology of breathing and its relevance for understanding several clinical syndromes that are not directly associated with respiratory disturbance. We present findings from three areas - panic disorder, functional cardiac disorder, and chronic pain - supporting respiratory factors as either markers, mediators, or etiological mechanisms for a number of physical symptoms. Recent technological advances allowing the ambulatory measurement of end-tidal partial pressure of $\mathrm{CO}_{2}\left(\mathrm{pCO}_{2}\right)$, and respiratory patterns have opened up new avenues for investigation and treatment of these disorders. Recent evidence from our laboratories indicates that subtle but consistent breathing anomalies are present in panic patients as opposed to other anxiety disorder or normal control groups. Contrary to previous models that emphasized outright hyperventilation as a key factor in anxiety disorders, this research focuses on respiratory instability and breathing behaviors such as sighs as signs of dysfunctional regulation of breathing. Finally, we discuss some treatment approaches based on the proposed mediational model that can be effective in the disorders mentioned above.

\begin{tabular}{ll}
\hline KARGER & @ 2000 S. Karger GmbH, Freiburg \\
Fax +497614520714 & Accessible online at: \\
$\begin{array}{l}\text { E-mail Information@Karger.de } \\
\text { www.karger.com }\end{array}$ & www.karger.com/journals/ver
\end{tabular}

Dr. Frank H. Wilhelm

Stanford University, VAPAHCS (116F-PAD)

3801 Miranda Ave., Palo Alto, CA 94304

Tel. +1 650 493-50 00 oder -652-42, Fax -49 01

E-mail fwilhelm@stanford.edu 


\section{Einleitung}

Die Atmung ist eine physiologische Funktion, die strategisch an der Schnittstelle zwischen Leib und Seele liegt. Normalerweise arbeitet die Atmung ohne bewusste Steuerung, sie kann aber auch - zumindest für kurze Zeit - unter willentliche Kontrolle gebracht werden, was zu tiefgreifenden körperlichen Veränderungen führen kann. Des weiteren ist die Atmung, anders als die meisten anderen physiologischen Systeme, einer detaillierten Introspektion zugänglich. Atmung ist wesentlich für die Lebenserhaltung, denn jedes Organsystem ist abhängig von einem adäquaten Gasaustausch. Die zentrale Bedeutung des Atmens bei menschlichen Gefühlen, der Gesundheit und dem Bewusstsein ist in Bereichen wie Literatur, Philosophie, Religion und Physiologie offensichtlich.

Überraschenderweise wurde der Atmung im Bereich der Psychophysiologie bis vor kurzem kaum Beachtung geschenkt. Als Reaktion auf dieses fehlende Interesse an der Atmung in der psychologischen Wissenschaft, wurde 1984 die International Society für the Advancement of Respiratory Psychophysiology gegründet [Timmons und Ley, 1994]. Mittlerweile haben Fortschritte im Verständnis der Physiologie der Atmung aufgezeigt, dass verschiedene homöostatische Systeme des menschlichen Körpers durch Atemmuster und den sich daraus ergebende Veränderungen der arteriellen $\mathrm{CO}_{2}-$ Konzentration beeinflusst werden. Aufbauend auf diesen Erkenntnissen, haben klinische Psychophysiologen erkannt, dass Wechselwirkungen zwischen emotionalen Zuständen, dem Atemverhalten (wie z.B. zu tiefes, schnelles oder unregelmäßiges Atmen, Seufzen, Gähnen oder Luftanhalten) und der Atmungsphysiologie als mögliche Mediatoren bei Störungen wie Asthma, Panikstörung, generalisierter Angststörung (GAS), Reizdarm, Hyperventilationssyndrom (HV-Syndrom) und funktionellen Herzbeschwerden eine Rolle spielen. Außerdem ist die optimierte Regulierung der Atmung bei Arbeitsund sportlichen Leistungen wichtig.

In dieser Übersicht präsentieren wir grundlegende Informationen zur Physiologie der Atmung, die für viele Psychotherapeuten nicht bekannt sein dürften. Wir geben einen Überblick über Befunde zur Rolle der Atmung bei der Entstehung und Aufrechterhaltung einer Reihe von Störungen sowie bei deren Behandlung, und schließen dabei unsere eigenen neuesten Forschungsergebnisse ein. Wie der Leser sehen wird, befinden wir uns hier im Anfangsstadium der Erstellung einer systematischen wissenschaftliche Datenbasis für dieses vielversprechende Gebiet.

\section{Physiologischer Hintergrund}

Die grundlegende Funktion des respiratorischen Systems besteht darin, die Organe mit Sauerstoff $\left(\mathrm{O}_{2}\right)$ zu versorgen, da dieser zur Energiegewinnung beim oxidativen Abbau der Nährstoffe benötigt wird. Das bei diesem Stoffwechsel entste-

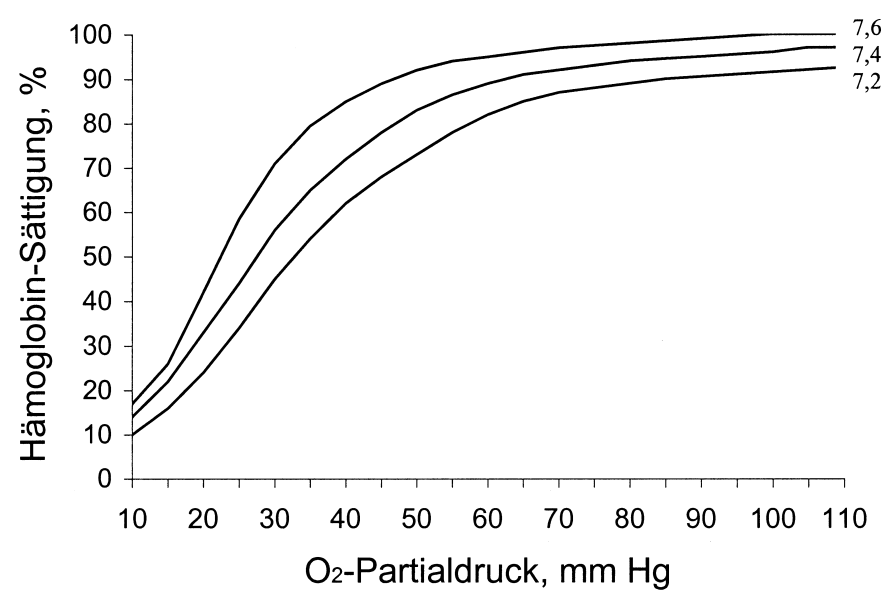

Abb. 1. Sauerstoff-Hämoglobin- $\mathrm{O}_{2}-\mathrm{Bindungskurven} \mathrm{und} \mathrm{Bohr-Effekt}$ (siehe Text). HV führt zu einer Verschiebung der Kurve nach links, wodurch das $\mathrm{O}_{2}$ stärker an das Hämoglobin gebunden wird. Dies kann unter bestimmten Bedingungen zu einer Beeinträchtigung der $\mathrm{O}_{2}$-Abgabe an das körperliche Gewebe führen. Zur Veranschaulichung sind die Unterschiede in den Kurvenverläufen für Blut-pH-Werte von 7,2 bis 7,6 leicht übertrieben dargestellt.

hende Abfallprodukt Kohlendioxid $\left(\mathrm{CO}_{2}\right)$ wird dann über die Lungen abgeatmet. Obwohl $\mathrm{CO}_{2}$ oft lediglich als ein Abfallprodukt angesehen wird, spielt es eine entscheidende Rolle bei der Aufrechterhaltung des Säure-Base-Gleichgewichts im inneren Milieu des Körpers.

Über orale oder nasale Atemwege aufgenommene Luft wird, während sie Luftröhre und Bronchien passiert, aufgewärmt, gereinigt und angefeuchtet, bevor sie die Lunge erreicht. In den Alveolen der Lunge findet der Gasaustausch statt. Die Alveolen haben sehr dünne Wände und breiten sich über eine enorme Oberfläche aus - im Durchschnitt $140 \mathrm{~m}^{2}$. Jedes Lungenbläschen ist von einer Vielzahl von Lungenkapillaren umgeben, dessen Wände ebenfalls sehr dünn sind. Der Gasaustausch findet durch diese Kapillarwände hindurch statt. Da die inhalierte Luft zu etwa $21 \%$ aus $\mathrm{O}_{2}$ und nur zu etwa $0,05 \%$ aus $\mathrm{CO}_{2}$ besteht (der Rest ist hauptsächlich Stickstoff), hat in den Alveolen das $\mathrm{O}_{2}$ einen sehr hohen $\mathrm{O}_{2}$-Partialdruck $\left(\mathrm{pO}_{2}\right)$ (etwa $105 \mathrm{~mm} \mathrm{Hg}$ ) und die Tendenz, durch die Wände der Alveolen und Kapillaren ins Blut zu diffundieren. Umgekehrt hat das $\mathrm{CO}_{2}$ in der alveolaren Luft einen geringen $\mathrm{CO}_{2}$-Partialdruck $\left(\mathrm{pCO}_{2}\right)$ (etwa $40 \mathrm{~mm} \mathrm{Hg}$ ), deshalb diffundiert gleichzeitig das $\mathrm{CO}_{2}$ aus dem venösen Blut, wo es einen höheren $\mathrm{pCO}_{2}$ hat (etwa $45 \mathrm{~mm} \mathrm{Hg}$ ), in die umgekehrte Richtung, wird von den Alveolen aufgenommen und anschließend ausgeatmet. Die ausgeatmete Luft ist hochgradig mit $\mathrm{CO}_{2}$ angereichert und hat am Ende der Ausatmung typischerweise einen $\mathrm{pCO}_{2}$ von etwa $40 \mathrm{~mm} \mathrm{Hg}$.

Das $\mathrm{O}_{2}$ wird im Blut hauptsächlich durch das Hämoglobin (ein Protein, in das ein Eisenatom eingebaut ist) der roten 
Blutzellen aufgenommen und zu den Zielorganen transportiert. Wie viel $\mathrm{O}_{2}$ transportiert wird, hängt von verschiedenen Faktoren ab, wie etwa dem Anteil des verfügbaren $\mathrm{O}_{2}$ in der inhalierten Luft, der Temperatur und dem pH-Wert des Bluts, der normalerweise bei 7,4 liegt. Die Beziehung zwischen dem $\mathrm{pO}_{2}$ im Blut und dem Prozentsatz an Hämoglobin-Sättigung (dem Anteil von $\mathrm{O}_{2}$ der schon vom Hämoglobin aufgenommen wurde) wird durch die Hämoglobin- $\mathrm{O}_{2}$-Bindungskurve dargestellt (Abb. 1). Bei einem normalen Blut-pH-Wert besitzt diese Kurve einen sehr steilen Anstieg bei einem $\mathrm{pO}_{2}$ zwischen 0 und $40 \mathrm{~mm} \mathrm{Hg}$ und flacht bei höheren Partialdrücken ab. Das Hämoglobin hat eine so große Affinität $\mathrm{zu} \mathrm{O}_{2}$, dass fast immer eine stabile Sauerstoffversorgung der Organe sichergestellt ist, selbst unter so verschiedenen Bedingungen wie körperlicher Anstrengung (wenn der $\mathrm{O}_{2}$-Bedarf hoch ist) oder in großen Höhen, wo sehr viel weniger $\mathrm{O}_{2}$ in der inhalierten Luft zur Verfügung steht. Unter normalen Bedingungen ist das Blut, wenn es die Lungen verlässt, zu etwa 97\% mit $\mathrm{O}_{2}$ gesättigt.

Das $\mathrm{CO}_{2}$ ist als Kohlensäure im Blut gelöst. Jede Veränderung der Konzentration des gelösten $\mathrm{CO}_{2}$ verändert damit den Blut-pH-Wert, was zu einer Verschiebung der HämoglobinBindungskurve führt (Abb. 1). Diese Verschiebung ist wohl als eine geniale «Erfindung» der Evolution anzusehen, denn sie spielt eine bedeutsame Rolle bei der Erhöhung der $\mathrm{O}_{2}$-Anreicherung des Bluts in den Lungen und später bei der Erleichterung der Freisetzung von $\mathrm{O}_{2}$ im Gewebe. «Dies wird als Bohr-Effekt bezeichnet und kann folgendermaßen erklärt werden: Wenn das Blut die Lungen passiert, diffundiert $\mathrm{CO}_{2}$ vom Blut in die Alveolen. Dies vermindert den $\mathrm{pCO}_{2}$ des Bluts und die Konzentration der $\mathrm{H}^{+}$-Ionen (und erhöht den pH-Wert) aufgrund der resultierenden Abnahme der Kohlensäure. Beide Effekte verschieben die Hämoglobin-Bindungskurve nach links und heben sie an. ... Deshalb wird der Anteil des $\mathrm{O}_{2}$ beträchtlich erhöht, der sich an das Hämoglobin bei einem bestimmten alveolaren $\mathrm{pCO}_{2}$ bindet und ermöglicht somit einen größeren $\mathrm{O}_{2}$-Transport zum Gewebe. Wenn dann das Blut die Kapillaren des Gewebes erreicht, tritt genau der gegenteilige Effekt ein. $\mathrm{CO}_{2}$, das vom Gewebe ins Blut übertritt, schiebt die Kurve nach rechts. So wird $\mathrm{O}_{2}$ leichter vom Hämoglobin verdrängt und liefert somit dem Gewebe $\mathrm{O}_{2}$ mit einem höheren Partialdruck als unter anderen Bedingungen.» [nach Guyton, 1995, S. 518].

HV, definiert als «Atmung, die die Erfordernisse des Stoffwechsels übersteigt» [Gardner, 1994], kann sich in Form eines dramatischen medizinischen Notfalls ereignen oder das Resultat einer subtileren Störung des normalen Atmens wie häufigen Seufzens oder einer vertieften Brustatmung sein. HV vermindert die $\mathrm{CO}_{2}$-Konzentration des Bluts und macht den $\mathrm{pH}$-Wert des Bluts geringfügig alkalischer (erhöht den $\mathrm{pH}$ Wert), was eine linksseitige Verschiebung der Hämoglobin$\mathrm{O}_{2}$-Bindungskurve verursacht (Abb. 1). Fried [1987] nimmt an, dass diese Verschiebung in Verbindung mit der Konstriktion zerebraler Arterien (ein anderer Prozess hervorgerufen durch die Alkalose), die $\mathrm{O}_{2}$-Freisetzung im Gehirn dahingehend verschlechtert, dass einige Gehirnfunktionen durch die resultierende Hypoxie beeinträchtigt werden. Andere Effekte der Alkalose sind eine Zunahme des intrazellulären $\mathrm{Ca}^{2+}$, der sympathikotonen neuronalen Katecholamine, des koronararteriellen Blutdrucks, des peripheren vaskulären Widerstands und kardialer Arrhythmien sowie eine Absenkung des extrazellulären $\mathrm{Ca}^{2+}$ und $\mathrm{K}^{+}$, das sich auf die neuronale Erregbarkeit auswirkt [Gilbert, 1999]. Da diese systemischen Veränderungen eine Reihe von körperlichen Symptomen hervorrufen können, liegt die Vermutung nahe, dass HV ein Bindeglied zwischen psychischen und physischen Zuständen bei klinischen Syndromen wie Angst oder psychosomatischen Störungen (gastroenterologische Störungen, Asthma, Bluthochdruck, Muskelverspannung usw.) ist.

Der menschliche Organismus hat verschiedene redundante Systeme entwickelt, um einen Anstieg des arteriellen $\mathrm{pCO}_{2} \mathrm{zu}$ begrenzen. Wenn der $\mathrm{pCO}_{2}$ über den normalen Grenzwert ansteigt, stimulieren arterielle und neuronale Mechanismen eine verstärkte Atemaktivität, die dann den $\mathrm{pCO}_{2}$ reduziert. Das trifft jedoch nicht zu, wenn $\mathrm{CO}_{2}$ unter den normalen Grenzwert abfällt. Es gibt nur wenige Ergebnisse, die für einen reflexähnlichen Mechanismus dieser Art sprechen, obwohl medulläre und kortikale Zentren wahrscheinlich HV dämpfen können. Ferner ist eine untere Grenze für einen normalen $\mathrm{pCO}_{2}$-Wert schwierig zu definieren.

Bevor wir die Ergebnisse diskutieren, die dafür sprechen, dass die Atmung ein wichtiges Bindeglied zwischen psychischen und physischen Prozessen darstellt, bedarf es der näheren Betrachtung der Möglichkeiten, respiratorische Parameter nichtinvasiv zu messen. Traditionelle medizinische Lungenfunktionsmaße wie maximale Atemstromstärke (peak expiratory flow, PEF), Atemwegswiderstand (respiratory resistance) oder 1-s-Ausatmungskapazität (forced expiratory volume, FEV) waren nicht von primärem Interesse für die respiratorische Psychophysiologie. Stattdessen richtete sich die Aufmerksamkeit auf den $\mathrm{pCO}_{2}$ am Ende der Ausatmung (endtidal $\mathrm{pCO}_{2} ; \mathrm{PetCO}_{2}$ ), die Atemfrequenz und die thorakale/abdominelle Dominanz. Man kann $\mathrm{PetCO}_{2}$ mit einem Kapnographen (auch Kapnometer genannt) messen. Dieses Gerät arbeitet typischerweise so, dass kontinuierlich eine Probe der ausgeatmeten Luft der Nase oder des Munds eingesaugt wird, mit einem Gas-Spektrometer der Gasinhalt analysiert wird und die resultierenden Werte digital und/oder graphisch dargestellt werden. Entscheidend ist der maximale Wert, der am Ende der Ausatmung erreicht wird (end-tidal), da dieser am besten die $\mathrm{CO}_{2}$-Konzentration im arteriellen Blut widerspiegelt. Die Werte werden normalerweise in $\mathrm{mm} \mathrm{Hg}$ oder Torr angegeben. Werte für gesunde Personen befinden sich typischerweise in einem Bereich zwischen 38 und $42 \mathrm{~mm} \mathrm{Hg}$. Ein alternatives $\mathrm{Maß}$ ist $\% \mathrm{PetCO}_{2}$ (hier ist 5,2\% typisch). Auch die Atemfrequenz wird oft angezeigt. Die Atemfrequenz lässt sich auch mit Hilfe eines einfachen Dehnungsgürtels um Thorax oder Abdomen, eines an der Nasenöffnung befestigten 
Temperatursensors, oder zweier EMG-Elektroden messen, die über an der Atmung beteiligten muskulären Strukturen (z.B. Trapezmuskel), die an der Atmung beteilig sind, angebracht werden. Darüber hinaus ist eine in der Forschung häufig benutzte Messmethode die induktive Plethysmographie $\left(\right.$ Respitrace $\left.^{\mathrm{TM}}\right)$. Hierbei wird mit Hilfe zweier in Bänder eingewobener, gewundener Drahtschleifen, die ein schwaches elektromagnetisches Feld erzeugen, die Fläche des Querschnitts durch Abdomen und Thorax gemessen. Bei adäquater Kalibrierung (durch Atmen in einen Plastikbehälter von genau abgemessenem Volumen) ermöglicht diese Methode eine kontinuierliche Messung des absoluten Atemzugvolumens in Litern.

Einige Forscher richteten ihre Aufmerksamkeit auf das Muster von $\mathrm{PetCO}_{2}$ im Zeitverlauf einzelner Atemzüge. Landis und Romano [1998] untersuchten kapnographische Wellenformen von drei klinischen Gruppen (Asthma, Angst/Panik und stressbezogene Störungen) und bei normalen Kontrollpersonen. Die klinischen Gruppen (vor allem Asthma und Angst/Panik) zeigten besonders viele atypische Wellenformen im Vergleich zur gesunden Kontrollgruppe, was als Hinweis auf subtile Störungen des Atemflusses gewertet wurde.

\section{Respiratorische Faktoren als Mediatoren und Marker von Störungen}

Die klinische Psychophysiologie versucht unter anderem herauszufinden, was genau die Verbindung zwischen psychologischen Variablen wie Stress, Angst, oder psychischen Störungen und körperlichen Symptomen herstellt. Ein physiologischer Mediator wird hierbei definiert als kausales Bindeglied zwischen psychischen und körperlichen Prozessen. Ein verwandtes Konzept ist das der physiologischen Marker. Ein Marker ist eine physiologische Abweichung, die nur bei einer bestimmten klinischen Population zu finden ist. Im Gegensatz zu Mediatoren stehen Marker nicht unbedingt in einem unmittelbaren kausalen Zusammenhang mit der Störung, die sie anzeigen. Marker können dabei hilfreich sein, die Entstehung für Angst und andere Störungen aufzuklären, indem sie einen «Endophänotyp» [Klein, 1998] aufzeigen. Der Versuchsaufbau der meisten unten besprochenen Studien erlaubt keine klare Unterscheidung zwischen Mediator und Marker, weshalb wir diese nicht getrennt behandeln.

Sowohl Mediatoren als auch Marker bieten die Möglichkeit, diagnostische und behandlungsrelevante Entscheidungen zu unterstützen. Insbesondere könnte die Entdeckung von Markern für Angststörungen zu teilweise neuen Zuordnungen von Patienten zu DSM-IV-Kategorien [American Psychiatric Association, 1994] führen. Ein Beispiel soll das verdeutlichen: Obwohl Patienten mit Symptomen einer typischen Panikstörung leicht identifiziert werden können, zeigen viele ängstliche Patienten Symptome, die untypisch sind. Diese Patienten werden möglicherweise fehldiagnostiziert, wenn man sich al- lein auf deren (manchmal retrospektiv verzerrten) Selbstberichte verlässt und Zuflucht zur Restkategorie «Angststörungen NNB» oder zur Kategorie «Angst und Depression gemischt» nimmt. Wenn ein Subtyp der Angststörungen des DSM-IV durch eine spezifische physiologische Abweichung charakterisiert werden kann und diese Abweichung wiederum direkt zu einer spezifischen Behandlung führt, sollte dies bessere Ergebnisse ermöglichen als eine unspezifischere kognitiv-behaviorale oder medikamentöse Behandlung.

Bis heute basieren Effektivitätsstudien für Angststörungen auf DSM-IV-Kategorien, die sich ausschließlich auf Selbstberichte stützen, die einem Interviewer mitgeteilt werden. Wie mittlerweile immer mehr erkannt wird, ist ein Selbstbericht über Symptome oftmals kein guter Zugang zu einer Störung, die physiologische Komponenten beinhaltet. Neben Erinnerungsfehlern, die bei der Erhebung retrospektiver Selbstberichte auftreten [Margraf et al., 1987], ist die enterozeptive Genauigkeit für Angst-Symptome wie z.B. den Herzschlag gering [Ehlers und Breuer, 1996], und die Selbstberichte von Symptomen unterliegen in hohem Maße situationalen, dispositionalen und motivationalen Faktoren [Pennnebaker, 2000]. Im Folgenden stellen wir Forschungsergebnisse dar, die die Bedeutung von respiratorischen Faktoren für Angst und verschiedene körperliche Beschwerden verdeutlichen.

\section{Angststörungen}

Die Suche nach Mediatoren und Markern für Angststörungen Schon seit Jahrzehnten wird postuliert, dass bei Angststörungen Abweichungen in der Atmung eine zentrale Rolle spielen, da Patienten oft von schweren Atembeschwerden während der Angstanfälle berichten. Es wurde mehrfach festgestellt, dass neben Herzrasen und Schwindelgefühlen die Kurzatmigkeit das am häufigsten berichtete Paniksymptom ist [Margraf et al., 1987; McNally et al., 1995]. Somit richtete sich bei der Suche nach physiologischen Mediatoren und Markern für die Panikstörung ein Hauptaugenmerk auf das respiratorische System. Diese Suche weiter einschränkend, weisen neuere Berichte darauf hin, dass die Panikstörung in zwei Subtypen unterteilt werden kann, solche mit und solche ohne bedeutsame respiratorische Symptome [Aronson und Logue, 1988; Briggs et al., 1993; Shioiri et al., 1996]. Beim respiratorischen Subtyp wurde festgestellt, dass dieser eine größere physiologische Reaktivität bei einer $\mathrm{CO}_{2}$-Inhalation [Biber und Alkin, 1999] und HV-Provokation [Hegel und Ferguson, 1997] aufweist. Bis dahin unterschied ein Großteil der Forschung in diesem Gebiet nicht zwischen diesen Subtypen, was die Effektgrößen für respiratorische Marker reduziert haben könnte und verschiedene inkonsistente Befunde erklären kann. Im Folgenden geben wir einen Überblick zu den Belegen für respiratorische Abweichungen bei Angststörungen und stellen aktuelle Ergebnisse unserer Forschung dar. Wir werden uns auf die Panikstörung konzentrieren, da sie angesichts der oft- 
mals verursachten Kosten eine wichtige Rolle innerhalb des Gesundheitssystems spielt und da es konvergierende Befunde gibt, die anzeigen, dass respiratorische Faktoren bei dieser Störung besonders wichtig sind. Die klinische Beobachtung legt nahe, dass Panikattacken auch bei sozialer und spezifischer Phobie auftreten. Somit wäre es nicht überraschend, wenn diese Diagnosen einige respiratorische Merkmale mit der Panikstörung teilen würden.

\section{Hyperventilation als Mediator und Marker}

Vom HV-Syndrom [Kerr et al., 1937] wurde angenommen, dass es einer Vielzahl psychosomatischer Beschwerden und Befindlichkeitsstörungen zu Grunde liegt. Seit der Einführung der Panikstörung als einer diagnostischen Kategorie wurden HV-Episoden besonders mit Panikattacken in Verbindung gebracht [Ley, 1985]. Es ist offensichtlich, dass sich beide Beschwerdebereiche überschneiden [Hornsveld und Garssen, 1997; Ley, 1985]. In ihrer einfachsten Form postuliert diese Betrachtungsweise, dass HV selbst die Befindlichkeitsveränderungen und Symptome hervorruft, die bei Angststörungen zu beobachten sind [Lum, 1987]. Dies erscheint berechtigt, da absichtlich hervorgerufene $\mathrm{HV}$ bei gesunden Personen zu einem Muster körperlicher und psychologischer Symptome führen kann, das dem von akuten Angstsymptomen ähnelt z.B. Kurzatmigkeit, Herzrasen, Schwindel, Schwäche oder Kribbeln in den Extremitäten.

Die empirische Befundlage unterstützt diese Perspektive nur teilweise. Absichtliche HV wurde verwendet, um Panikattacken im Labor zu provozieren [z.B. Gorman et al., 1984], und andere Panikprovokationen bei Angstpatienten führen typischerweise zu Hypokapnie (geringem $\mathrm{pCO}_{2}$ ) [z.B. Gorman et al., 1988b]. Hypokapnie zeigte sich wiederholt als ein Unterscheidungsmerkmal zwischen Panikpatienten und einer Vergleichsgruppe während der Baseline-Phasen [Hegel und Ferguson, 1997; Papp et al., 1997]. Rapee [1986] fand dass während einer Ruhephase vor einer absichtlichen HV Panikpatienten geringere $\mathrm{PetCO}_{2}$ und höhere Herzraten aufwiesen als Patienten mit GAS. Munjack et al. [1993] berichteten, dass der venöse $\mathrm{pCO}_{2}$ in Ruhe (ohne die Erwartung eines Stressors wie HV) bei Panikpatienten geringer war als bei GAS-Patienten und dass bei GAS-Patienten $\mathrm{PetCO}_{2}$ geringer war als bei einer Kontrollgruppe. Eine unserer neueren Analysen [Wilhelm et al., 2000a] bestätigte, dass Patienten mit einer Panikstörung im ruhigen Sitzen niedrigere $\mathrm{PetCO}_{2}$ hatten als GASPatienten oder eine Kontrollgruppe. In einer anderen Studie hatten sowohl Patienten mit Panikstörung als auch andere Angstpatienten niedrigere PetCO $\mathrm{C}_{2}$-Ruhewerte als eine Kontrollgruppe, und die Angstgruppen unterschieden sich nicht voneinander [van den Hout et al., 1992]. Eine Studie lieferte Hinweise für eine chronische HV bei Panikpatienten vor der Behandlung und eine Normalisierung nach der Behandlung [Salkovskis et al., 1986b]. Einige wenige Studien konnten keine Unterschiede im PetCO $_{2}$ zwischen Panikpatienten und Kontrollgruppen finden [Holt und Andrews, 1998; Woods et al., 1986]. Eine verlangsamte Erholung der $\mathrm{PetCO}_{2}$-Werte nach absichtlicher HV im Vergleich zu gesunden Kontrollgruppen wurde wiederholt bei Panikpatienten gefunden [Gorman et al., 1988a; Maddock und Carter, 1991]. Aber wiederum konnten verschiedene Studien diese langsame Erholung nicht bestätigen [Hegel und Ferguson, 1997; Rapee, 1986], was auf die kürzere HV ( $<3 \mathrm{~min})$ oder Erholungszeiten $(<5 \mathrm{~min}) \mathrm{zu}-$ rückführbar sein könnte.

Entgegen dieser Befunde haben neuere Ergebnisse die Bedeutung der HV für die Panikstörung und andere Angststörungen in Frage gestellt. Erstens ist absichtliche HV eine relativ schwache Panikprovokation im Vergleich zu anderen biologischen Tests wie z.B. die Inhalation von $\mathrm{CO}_{2}$ oder die Infusion von Natriumlaktat [Gorman et al., 1988a; Gorman et al., 1994; Papp et al., 1997]. Zweitens waren nur einige spontane Panikanfälle von einer Hypokapnie begleitet (3 von 5 [Hibbert und Pilsbury, 1988]; 7 von 15 [Hibbert Pilsbury, 1989]; 1 von 24 [Garssen et al., 1996]), wenn das Niveau des arteriellen $\mathrm{pCO}_{2}$ bei Panikpatienten kontinuierlich unter Verwendung von transkutanen Techniken außerhalb des Labors erhoben wurde [Garssen et al., 1994]. Demnach scheinen die ambulanten Messergebnisse die Hypothese teilweise zu widerlegen, dass HV Panikanfälle verursacht oder etwas zu deren Symptomen oder Schwere beiträgt. Dennoch können diese Ergebnisse aus zwei Gründen nicht als abschließend betrachtet werden: Erstens hat die ambulante transkutane $\mathrm{pCO}_{2}$-Messung ihre Grenzen hinsichtlich der Widerspiegelung von $\mathrm{pCO}_{2}$-Veränderungen im Blut; z.B. führt die etwa 2-minütige Messverzögerung bedingt durch die langsame Diffusion des $\mathrm{CO}_{2}$ durch die Haut zu einer Glättung der $\mathrm{pCO}_{2}$-Kurven, so dass weniger intensive oder kurze HV-Episoden übersehen werden können. Zweitens legt die große Variation der oben erwähnten Studien in den Anteilen von Panikanfällen, die mit Hypokapnie einhergehen, nahe, dass sich die Stichproben im Hinblick auf ihren Anteil an Patienten des respiratorischen Subtyps unterscheiden. Dies kann auch einige der oben erwähnten sich widersprechenden Ergebnisse zur Baseline-Messung und Erholung des PetCO ${ }_{2}$-Niveaus nach $\mathrm{HV}$ erklären.

Eine von uns durchgeführte Studie über Exposition in vivo bei Flugphobikern [Wilhelm und Roth, 1998] zeigte, dass sowohl Patienten als auch Kontrollpersonen während eines kurzen 12-minütigen Fluges eine erhöhte Atemfrequenz und ein erhöhtes Minutenvolumen aufwiesen, wobei nur die Patienten Atemanomalien in Form von ausgeprägten Unterbrechungen der Atmung zeigten. In einer noch laufenden ambulatorischen Studie mit Autofahrphobikern [Alpers et al., 2000] untersuchen wir nun die Effekte einer längeren und wiederholten Exposition auf die Atmung. Die vorläufigen Ergebnisse bestätigen, dass Exposition in vivo akute Veränderungen der respiratorischen Physiologie verursacht. Das Autofahren führte zu einer ungefähr gleich starken Erhöhung der Atemfrequenz bei Patienten und Kontrollpersonen, während das Atemzugvolumen nur bei den Patienten erhöht war und damit zu einem größeren Minutenvolumen und zu einer Ver- 


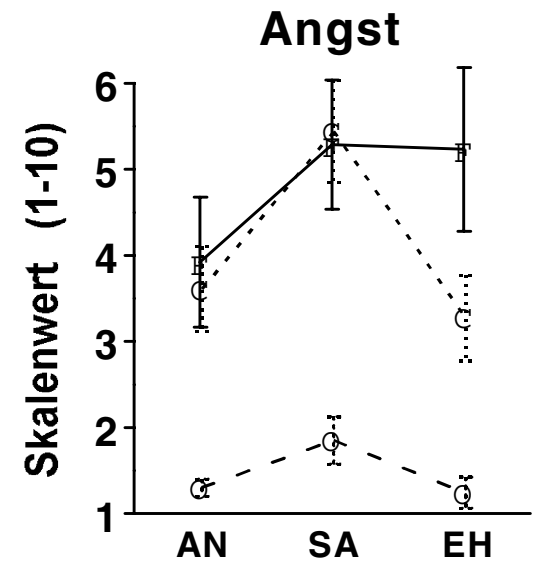

a

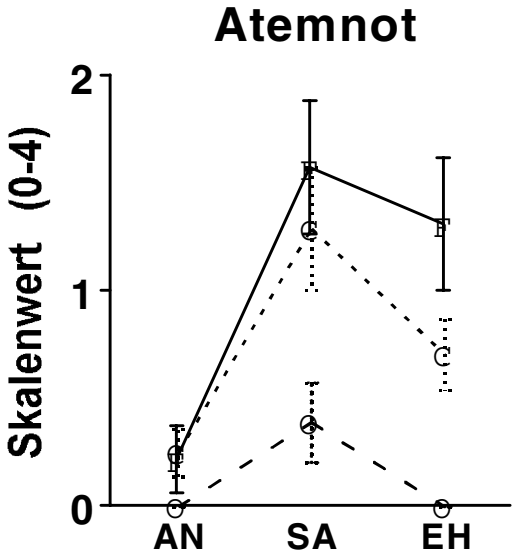

b
Atembewusstsein

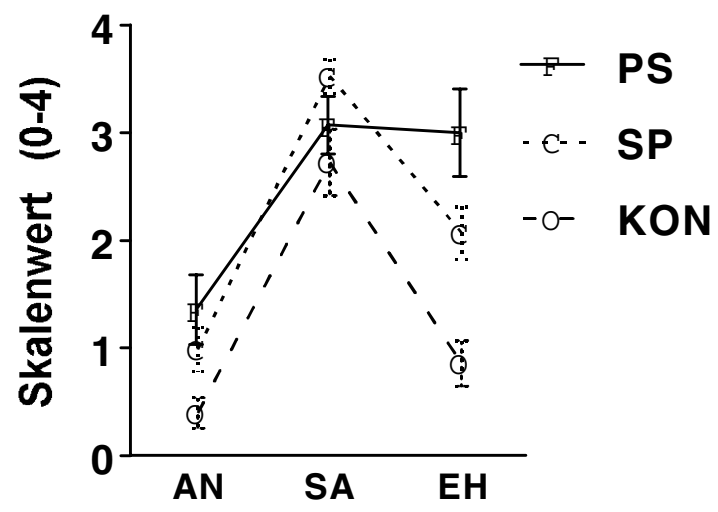

Abb. 2. Mittelwerte und Standardfehler für die Selbsteinschaeätzungsmaße a Angst, b Atemnot, und c Atembewusstsein im Verlauf des Experiments für Patienten mit Panikstörung (PS), mit Sozialphobie (SP) und für Kontrollpersonen (KON). AN = Ausgangsniveau; SA = schnelles Atmen; EH = Erholung.

ringerung des $\mathrm{PetCO}_{2}$ bei den Patienten führte (Baseline 35 $\mathrm{mm} \mathrm{Hg}$; nach $30 \mathrm{~min}$ Exposition $28 \mathrm{~mm} \mathrm{Hg}$ ). Die Patientenberichte über eine Verringerung der Angst nach einigen Expositionssitzungen waren von synchronen Veränderungen der physiologischen Parameter in Richtung der Werte von Kontrollpersonen begleitet. Diese Studien zeigen, dass HV während einer Angsttherapie auftreten kann, und dass ambulatorische Messungen zu einem Verständnis der bei einer Verhaltenstherapie ablaufenden Prozesse beitragen können.

Daten einer neueren, noch nicht publizierten Studie aus unserem Labor weisen darauf hin, dass die langsame Erholung des $\mathrm{PetCO}_{2}$ nach $\mathrm{HV}$ bei Patienten mit Panikstörung nicht auf das respiratorische System beschränkt ist, sondern mit einer Vielzahl an autonomen und subjektiv erlebten Abweichungen einhergeht. Wir untersuchten respiratorische, autonome und subjektive Reaktionen bei 14 Patienten mit Panikstörung, $24 \mathrm{~Pa}$ tienten mit einer sozialen Phobie und 24 Kontrollpersonen während 6 Durchgängen von 1-minütigem, schnellem und tiefen Atmen (unterbrochen von 1-minütigen Pausen), gefolgt von 3 min schnellem und tiefem Atmen und 10 min Erholungszeit (Abb. 2 und 3). Der Atemrhythmus von 18 Zügen/min wurde durch einen Ton vorgegeben und die Atemtiefe durch ein visuelles Feedback. Den Patienten wurde als Ziel vorgegeben, ein $\mathrm{PetCO}_{2}$ von $20 \mathrm{~mm} \mathrm{Hg}$ zu erreichen. Während des schnellen Atmens erreichten alle drei Gruppen dieselben Werte für Atemfrequenz, Minutenvolumen und $\mathrm{PetCO}_{2}$. Dennoch berichteten Patienten mit Panikstörung und sozialer Phobie im Vergleich zur Kontrollgruppe häufiger über Angst, und ihr Gefühl der Atemnot erhöhte sich relativ zur Baseline stärker (Abb. 2). Die Hautleitfähigkeit (ein Maß für Aktivierung des sympathischen Nervensystems) nahm bei den Patienten mit Panikstörung über die 6 wiederholten Phasen des schnellen Atmens langsamer ab (Abb. 3a). Am Ende der 10-minütigen Erholungszeit berichteten Panikpatienten erhöhte Werte für Angst, Atemnot, Atembewusstsein sowie Angst vor Atemnot (nicht dargestellt), und die Normalisierung ihrer Werte für PetCO 2 , Herzrate und Hautleitfähigkeit war im Vergleich zu den anderen Gruppen vermindert (Abb. 3a-c). Diese Ergebnisse zeigen, dass Patienten mit Panikstörung und sozialer Phobie bei einem vergleichbaren Ausmaß an Hypokapnie über mehr Missempfindungen berichten als Kontrollpersonen. Panikpatienten unterscheiden sich von Sozialphobikern und Kontrollpersonen darin, dass sie nach einer HV eine verlangsamte symptomatische und physiologische Erholung haben.

\section{Respiratorische Variabilität als Mediator und Marker}

Seit es die ersten Geräte zur exakten Messung der kontinuierlichen Atmung gibt, wurde berichtet, dass ängstliche Personen unregelmäßigere Atemmuster aufweisen als Kontrollprobanden. Geräte zur Ableitung eines Spirogramms erlaubten erstmals die genaue Erfassung des Atemzugvolumens. Auf diese Weise wurde gezeigt, dass «psychoneurotische» Patienten häufig seufzen [Finesinger, 1943]. Anhand der Häufigkeit von Seufzern (gemessen mittels induktiver Plethysmographie) konnten chronisch ängstliche Patienten von Patienten mit Asthma, chronisch obstruktiver Lungenerkrankung, restriktiver Lungenfunktionsstörung und primärer pulmonaler Hypertonie unterschieden werden [Tobin et al., 1983]. Es wurde beobachtet, dass ängstliche Personen, die häufig seufzen, eine normale totale Lungenkapazität, jedoch eine niedrigere Vitalund eine höhere Residualkapazität aufweisen [Aljadeff et al., 
1993]. Laktat-Infusionen, die besonders bei Patienten mit $\mathrm{Pa}$ nikstörung zum Angstanstieg führen, verstärkten bei diesen auch das Seufzen [Schwartz et al., 1996].

Seufzer werden häufig als Atemzüge quantifiziert, deren Atemzugvolumen das Doppelte der vor- und nachfolgenden Atemzüge überschreitet. Dieser wissenschaftliche Standard der Quantifizierung ist nicht gleichzusetzen mit dem umgangssprachlichen Verständnis von Seufzern, worunter typischerweise ein oft unbewusst ausgeübter tiefer Atemzug begleitet von einem klagenden Laut beim Ausatmen verstanden wird. In verschiedenen Studien wurden Seufzer nicht als distinkte Ereignisse erfasst. Statt dessen wurde die intraindividuelle Variabilität des Atemzugvolumens gemessen, indem statistische Kennwerte wie Standardabweichung, Variationskoeffizient oder mittlere quadrierte sukzessive Differenz (von-NeumannStatistik) verwendet wurden. In unseren Messungen sind die Korrelationen zwischen diesen unterschiedlichen Parameter hoch.

Abelson et al. [1996a] evaluierten den panikerzeugenden Effekt der atmungsstimulierenden Substanz Doxapram bei 16 Patienten mit Panikstörung und 16 parallelisierten Kontrollprobanden. Die Hälfte der Probanden in jeder Gruppe erhielt Instruktionen, die darauf abzielten, die Panikreaktion zu reduzieren, indem die Harmlosigkeit der Symptome betont wurde. Diese kognitive Intervention führte zu einer substantiell verringerten HV-Reaktion der Patienten. Eine erneute Datenanalyse [Abelson et al., 1996b] zeigte, dass die Patienten im Vergleich zur Kontrollgruppe in allen Phasen des Experiments (Baseline, Doxapram-Provokation, Erholung) erhöhte Werte der Atemzugvolumen-Variabilität aufwiesen. Weder die kognitive Intervention noch die mit Doxapram induzierte HV rief signifikante Veränderungen dieser Atmungsunregelmäßigkeit hervor. Die Patienten zeigten auch erhöhte Werte für die Atemfrequenz-Variabilität, wenn auch nicht so ausgeprägt und konsistent.

Stein et al. [1995] untersuchten das nächtliche Atmungsmuster von 14 Patienten mit Panikstörung und 14 parallelisierten Kontrollprobanden, um festzustellen, ob die Patienten mit $\mathrm{Pa}-$ nikstörung auch Atmungsunregelmäßigkeiten aufweisen zu Zeiten, in denen keine bewussten kognitiven Prozesse am Angsterleben beteiligt sind. Auf Atemanomalien bei den Patienten mit Panikstörung während des Schlafs wiesen einerseits die höhere Variabilität des Atemzugvolumens und andererseits die höhere Frequenz der Atmungspausen (mit einer Länge von 5 bis $10 \mathrm{~s}$ ) während des REM-Schlafes hin. Bystritsky et al. [2000] verglichen die physiologischen Reaktionen von 42 Patienten mit Panikstörung mit den Reaktionen von 25 Kontrollpersonen während einer Baseline-Messung, einer $5 \%$ igen $\mathrm{CO}_{2}$-Inhalation und einer Erholungsphase. Zwölf Panikpatienten erlebten während der $\mathrm{CO}_{2}$-Inhalation Angstanfälle, und diese wiesen eine größere Atmungsvariabilität auf, was durch die Länge und Anzahl der Atmungspausen und der Variabilität der Atemfrequenz quantifiziert wurde. In Übereinstimmung mit diesen Studien unterschied sich die Atem-

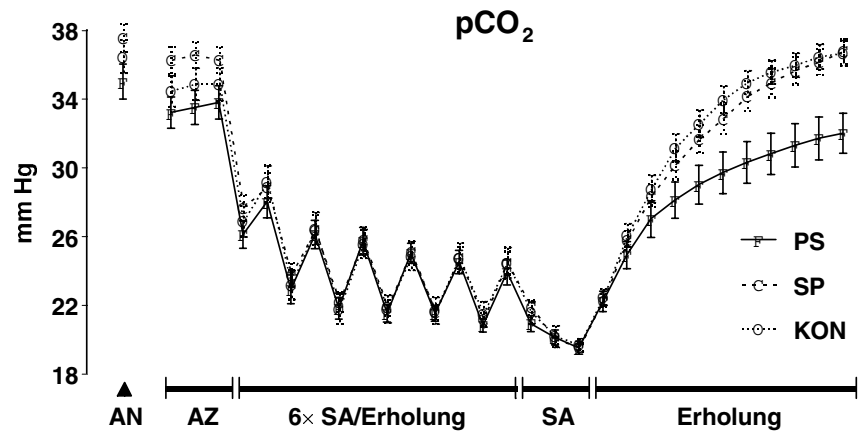

a

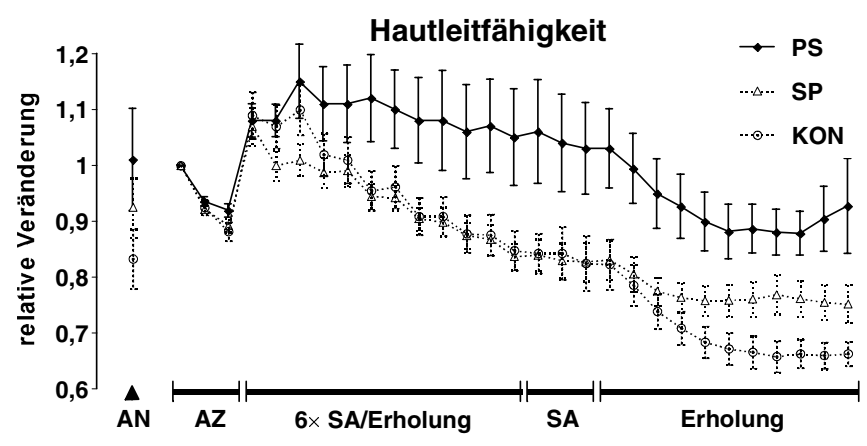

b

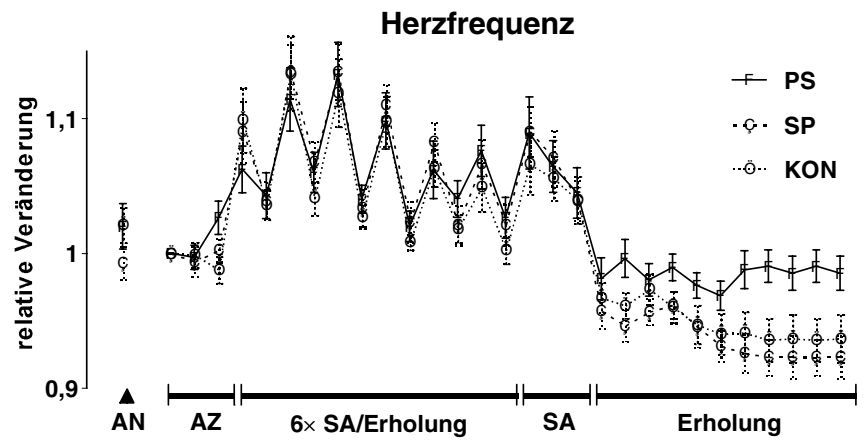

C

Abb. 3. Einminütige Mittelwerte und Standardfehler für die physiologischen $\mathrm{Maße}$ a $\mathrm{pCO}_{2}$, b Hautleitfähigkeit (zur besseren Veranschaulichung der Effekte ausgedrückt als relative Veränderung in Bezug zum Beginn der Antizipationsphase), und c Herzrate (auf die gleiche Weise normalisiert) im Verlauf des Experiments für Patienten mit Panikstörung (PS), mit Sozialphobie (SP) und für Kontrollpersonen (KON). AN = Ausgangsniveau; $\mathrm{AZ}=$ Antizipation; $\mathrm{SA}=$ schnelles Atmen.

zugvolumen-Variabilität während einer Baseline-Messung vor $\mathrm{CO}_{2}$-Inhalation auch bei Kindern und Jugendlichen mit Angststörungen von der bei psychisch gesunden Kindern [Pine et al., 1998]. 

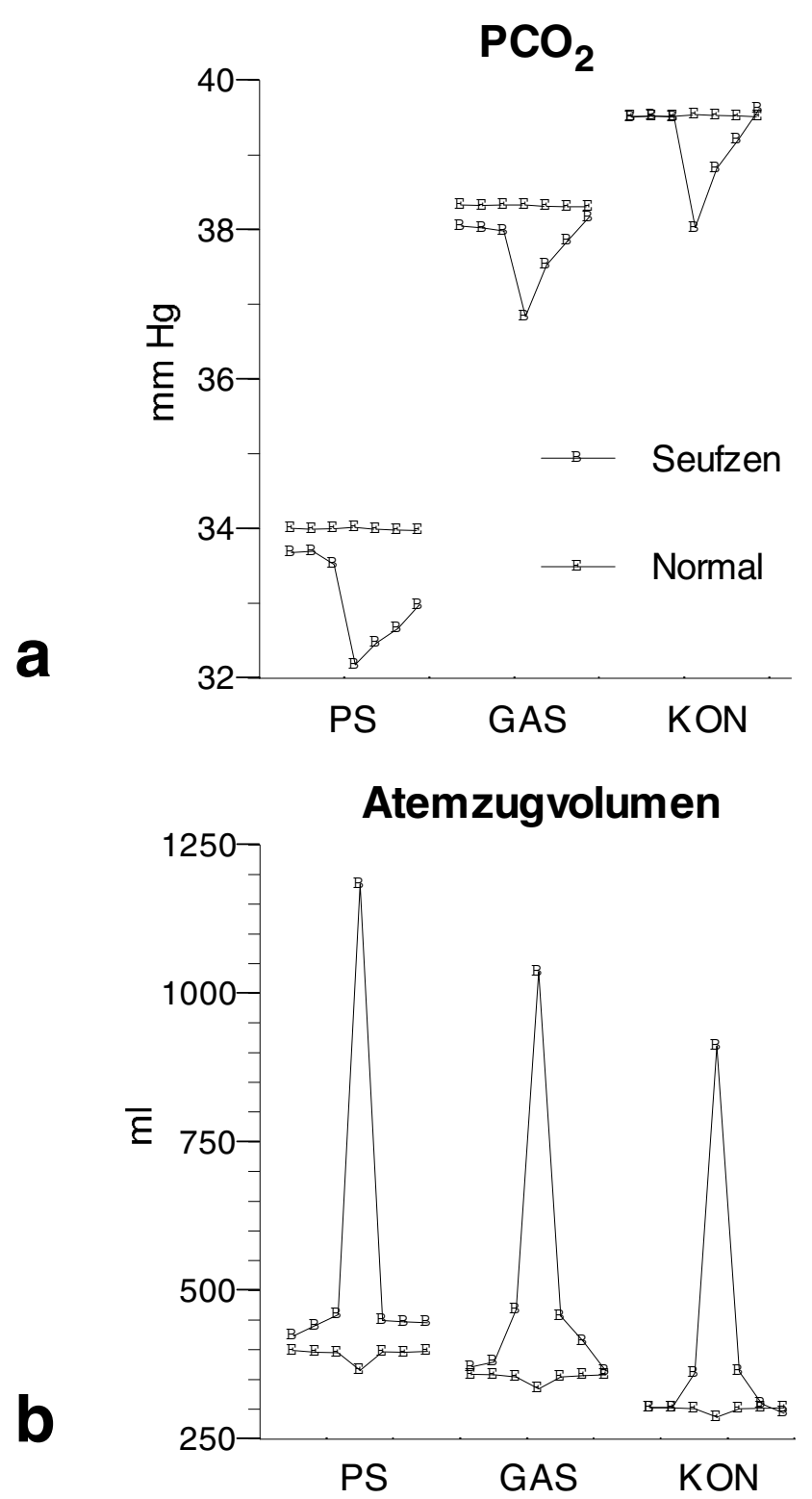

Abb. 4. Mittelwerte für $\mathbf{a} \mathrm{pCO}_{2}$ und $\mathbf{b}$ Atemzugvolumen für Patienten mit Panikstörung (PS), mit GAS und für Kontrollpersonen (KON). Dargestellt sind die durch Ensemble-Mittelung gewonnenen Werte für Atemzüge vor, während, und nach Seufzern. Zum Vergleich sind entsprechende Werte für Nicht-Seufzer («Normal») dargestellt.

Um die Atemzugvolumen-Variabilität quantitativ besser zu erfassen, führten wir in neueren Untersuchungen Frequenzband-Analysen durch. Diese Methode ist, um die oszillierende Variabilität innerhalb verschiedener Frequenzbänder zu beschreiben, genauer als das in den oben zitierten Studien verwendete herkömmliche Messverfahren. Wir fanden eine größere Atemzugvolumen-Variabilität innerhalb des Spektralbands von 0,004 bis $0,14 \mathrm{~Hz}$ bei Patienten mit Panikstörung verglichen mit GAS-Patienten oder Kontrollprobanden während einer Baseline-Messung [Wilhelm et al., 2000a]. Diese
Variabilität lässt sich nicht gänzlich durch die Seufzer (wie oben definiert) erklären. Selbst nach Ausschluss der Atemzüge mit Seufzen und deren Ersetzung durch interpolierte Werte, wiesen die Patienten mit Panikstörung noch eine größere Atemzugvolumen-Variabilität auf als die Kontrollgruppen. Das Interesse der beschriebenen Studie galt den spontanen Fluktuationen verschiedener biologischer Prozesse, die bei vollständigen Panikattacken relevant sind. Wir nahmen an, dass diese in schwächerer Ausprägung (als möglicherweise nicht wahrgenommene «Mini-Panikanfälle») auch unter den Ruhebedingungen des Experimentes auftreten sollten. Kardiovaskuläre, respiratorische und elektrodermale Variablen kamen aufgrund ihres Zusammenhangs mit Angst in Frage. Überraschenderweise wiesen jedoch nur die Atmungsparameter Gruppenunterschiede auf. Die Fluktuationen von kardiovaskulären und elektrodermalen Variablen, die man bei instabilen Aktivierungszuständen des autonomen Nervensystems erwarten könnte, waren in den Gruppen nicht unterschiedlich ausgeprägt.

In der oben bereits beschriebenen HV-Studie (Wilhelm et al., noch nicht publizierte Daten) beobachteten wir bei Patienten mit Panikstörung auch einen Variabilitätsanstieg des Atemzugvolumens (quantifiziert durch Frequenzband-Analyse oder Seufzerfrequenz) in der Erholungsphase nach der HV. Da der niedrige $\mathrm{PetCO}_{2}$ bei Patienten mit Panikstörung nicht mit einem erhöhten Minutenvolumen einher ging, scheint das seufzende Atmen für die verzögerte Erholung von der Hypokapnie, die durch die willentliche HV hervorgerufen wurde, verantwortlich zu sein.

Um die Bedeutung der Seufzer für die Atemregulation bei Patienten mit Panikstörung weiter zu untersuchen, führten wir eine genaue Analyse der Atmungsvariabilität während 30-minütigen ruhigen Sitzens durch [Wilhelm et al., 2000b]. Überraschenderweise konnte der individuelle $\mathrm{PetCO}_{2}$ besser durch die Häufigkeit von Seufzern als durch das Atemzugvolumen vorhergesagt werden. Die Auswertung der gemittelten Werte der Atemzüge, die Seufzern vorausgehen und nachfolgen (Ensemble-Mittelung), zeigte, dass in allen Gruppen der $\mathrm{PetCO}_{2}$ vor dem Auftreten von Seufzern reduziert und das Atemzugvolumen erhöht war (Abb. 4). Die Tiefe der Seufzer war bei den Patienten mit Panikstörung größer als bei den Probanden der Kontrollgruppen. Unmittelbar nach einem Seufzer kehrten der $\mathrm{PetCO}_{2}$ und das Atemzugvolumen bei Patienten mit Panikstörung nicht so schnell auf das Normalniveau zurück wie bei den Probanden der Kontrollgruppen. Diese Ergebnisse weisen darauf hin, dass die Hypokapnie bei Patienten mit Panikstörung mit ihrem häufigen Seufzen verbunden ist.

\section{Grenzen der Laborforschung zu Mediatoren und Markern}

Aus den vorangegangenen Abschnitten geht hervor, dass Atmungsstörungen wahrscheinlich eine wichtige vermittelnde Rolle bei der Entstehung von Angstsymptomen zukommt. Dagegen ist weniger klar, in welchem Ausmaß die zitierten 
Ergebnisse auf die durch den experimentellen Kontext ausgelöste Zustandsangst (state anxiety) zurückzuführen sind und in welchem Ausmaß sie überdauernde Charakteristika von Angstpatienten (trait characteristics) widerspiegeln. In einigen der Laborstudien erschienen die Atmungsanomalien nur während der Angstprovokation. Dies könnte als Indikator für den «State»-Effekt aufgefasst werden. Ruft die Provokation jedoch nur in einer diagnostischen Gruppe Angst hervor, könnte es sich um eine Interaktion aus «State»- und «Trait»Faktoren handeln.

In nur wenigen der zitierten Studien [Hegel und Ferguson, 1997; Holt und Andrews, 1998; Munjack et al., 1993; Rapee et al., 1992; van den Hout et al., 1992; Wilhelm et al., 2000a, 2000b] wurde untersucht, ob Atmungsanomalien spezifisch für eine oder mehrere vergleichbar ängstliche Patientengruppe sind (z.B. Panikstörung vs. GAS). Die Ergebnisse dieser Studien unterstützen (Ausnahme: [van den Hout et al., 1992]) die Annahme, dass die Atmungsdysregulation einen physiologischen Marker der Panikstörung darstellt, auch wenn andere Angststörungen ähnliche Anomalien in geringerem Ausmaß aufweisen.

Laborstudien können nur begrenzt solche «Trait»-Charakteristika demonstrieren, die bei Abwesenheit von Angst anhalten. Die Baseline-Werte spiegeln kaum prototypische Normalzustände wider, da Patienten mit Angststörungen oft unter Laborbedingungen besonders ängstlich sind. In den oben genannten Studien wurde das Baseline-Niveau durch wenigstens einen von drei Faktoren beeinflusst: vorangegangene Stressoren (z.B. Anfahrt zum Krankenhaus, andere klinische Tests), die experimentellen Untersuchungsbedingungen bei Erfassung der Baseline (z.B. wie invasiv die Geräte zur Atmungsmessung sind oder durch Einengungsgefühle innerhalb eines kleinen Untersuchungsraums) und die Furcht der Probanden vor nachfolgenden Tests (antizipatorische Angst).

Um die «State-trait-Frage» zu klären, sind ambulante Erhebungen in verschiedenen Settings nützlich. Solche Studien sind jedoch selten. Ein beachtenswertes Beispiel stellt die Studie der Forschungsgruppe um Klein [Martinez et al., 1996] dar, die ein tragbares Gerät zur 24-stündigen Atmungserfassung einsetzte. Das Atemzugvolumen (gemittelt über 2-minütige Intervalle) wies bei Patienten mit Panikstörung eine größere Variabilität auf als bei den parallelisierten Kontrollpersonen. Leider war es mit der eingesetzten Messmethode nicht möglich, die Werte pro Atemzug zu registrieren und auszuwerten. Die Phasen mit Angst und Panikanfällen waren eher durch Anstiege des Atemzugvolumens als durch Anstiege der Atemfrequenz charakterisiert. Die Ergebnisse weisen darauf hin, dass Patienten mit Panikstörung im Verlauf des Tages starke Schwankungen ihrer Angst erleben und dass diese von minutenlangen Veränderungen des Atemzugvolumens begleitet werden - zusätzlich zu der unter Laborbedingungen beobachteten Dysregulation bei der Erfassung einzelner Atemzüge.

\section{Funktionelle Herzbeschwerden}

Eine weiterer Beschwerdebereich, bei dem Atmungsanomalien möglicherweise eine vermittelnde Rolle bei der Symptomentstehung zukommt, betrifft die funktionellen Herzbeschwerden (FHB). FHB werden als Brustschmerzen oder kardiale Missempfindungen definiert, bei denen kein Hinweis auf eine Herzerkrankung vorliegt. $\mathrm{Zu}$ den typischen Symptomen zählen Brustschmerz, Arrhythmien, Kurzatmigkeit, Herzklopfen, Schwindel, Zittern, Schwitzen und Parästhesien. Wheatley [1975] schätzte, dass 50-90\% der FHB-Fälle mit einem HV-Syndrom in Verbindung stehen. Einige Autoren haben auch auf die Überschneidung von Panikstörung und FHB hingewiesen [Bass et al., 1988; Maddock et al., 1998]. Die Symptome dieser Störungen sind sich sehr ähnlich. Von daher ist denkbar, dass ihnen teilweise die gleichen pathophysiologischen Mechanismen zu Grunde liegen.

Es wurde angenommen, dass den FHB-Symptomen Störungsmechanismen wie z.B. Konstriktion und Spasmen der Herzkranzgefässe und Sauerstoffunterversorgung des Herzmuskels [Fujii et al., 1988; Yasue et al., 1989; Yu et al., 1959], erniedrigter parasympathischer Tonus [George et al., 1989], Sinustachykardie, Verringerung der Reizleitungszeit, reduzierter zerebraler Blutfluss [Kennealy et al., 1980] und Hypophosphatämie [Wheatley, 1975] zu Grunde liegen. DeGuire et al. [1992] fanden bei einer Gruppe mit FHB vor Behandlungsbeginn einen erniedrigten $\mathrm{PetCO}_{2}$ und eine erhöhte Atemfrequenz. Die Messung von Atmungsvorgängen hat sich für die Diagnostik und Behandlung dieser Patienten als sinnvoll erwiesen (siehe «Behandlungsaspekte»). Bass et al. [1988, S.88] stellten fest: «Beim Erleben von nichtkardialem Brustschmerz interagieren möglicherweise chronische Anomalien der Atmungskontrolle mit Einstellungsfaktoren.»

\section{Chronischer Schmerz}

Schon seit langem ist bekannt, dass Patienten während des Schmerzerlebens oft über Kurzatmigkeit klagen. Auch wurde gezeigt, dass Schmerz von Veränderungen der Atmung begleitet wird, welche wahrscheinlich durch einen verstärkten Atemantrieb verursacht werden [Nishino et al., 1999]. Glynn [1981] konnte zeigen, dass Patienten mit chronischen Schmerzen auf eine Schmerzblockade mit einem signifikanten Anstieg des $\mathrm{PetCO}_{2}$ reagierten, obwohl der $\mathrm{pH}$-Wert im Blut normal war. Dies macht es wahrscheinlich, dass eine über längere Zeit entwickelte biochemische Kompensation der HV-induzierten Veränderung des Blut-pH-Werts vorliegt. «Das deutet darauf hin, dass anhaltender Schmerz von chronischer HV begleitet wird und eine Schmerzabnahme mit einer Reduktion der Ventilation einher geht» [Glynn, 1981, S. 201]. Es gibt biochemische Befunde, die eine HV-induzierte Schmerzlinderung auf die Aktivität des endogenen Opiatsystems und des beim Schmerzempfinden beteiligten adrenergen Systems zu- 
rückführen [Ide et al., 1994a, 1994b]. Möglicherweise stellt die HV bei sowohl sensorisch als auch emotional quälendem Schmerz kurzfristig einen adaptiven Prozess mit eher negativen Langzeitkonsequenzen dar [Conway, 1994]. Viele Kliniker, auch einer der Autoren dieses Artikels (RG), hatten die Gelegenheit, bei Hunderten von Patienten mit chronischen Muskelschmerzen den $\mathrm{PetCO}_{2} \mathrm{zu}$ messen und stellten dabei überwiegend erniedrigte $\mathrm{pCO}_{2}$-Werte fest [z.B. Timmons und Ley, 1994].

\section{Behandlungsaspekte}

\section{Effektivität von Atemtraining}

Vor dem Hintergrund der geschilderten Befunde sollten Therapien, die direkt auf ungünstige Atemmuster einwirken, ein sinnvoller Behandlungsansatz sein. Atemtraining ist ein Baustein in einigen verhaltenstherapeutisch orientierten Behandlungsprogrammen, in den meisten meditativen Ansätzen (z.B. «Mindfulness»-Meditation) und in körperorientierten Therapien. Paradoxerweise gibt es trotz ihrer weitverbreiteten klinischen Verwendung nur begrenzte systematische Effektivitätsüberprüfungen für das Atemtraining oder das $\mathrm{PetCO}_{2}$-Feedback [Bass, 1994]. Studien, die eine Wirksamkeit von Atemtherapien nachweisen konnten, wurden kritisiert, weil deren Erfolg auch durch rein kognitive Faktoren erklärt werden könnte [de Ruiter et al., 1989; Salkovskis et al., 1986a]. Bass [1994] gibt einen kurzen Überblick über die Kontroverse zur therapeutischen Bedeutung von Atemtrainings. Tatsächlich ist es wahrscheinlich unmöglich, Patienten Atemtechniken zu vermitteln, ohne dass auch kognitive Bewertungsvorgänge verändert werden [Ley, 1991].

Creager und Gevirtz [2000] beendeten kürzlich eine Studie, bei der eine kognitiv-behaviorale Standardtherapie für Patienten mit Panikstörung mit einem Atemtraining mittels Kapnographie-Feedback verglichen wurde. Beide Therapiegruppen wiesen deutliche Verbesserungen auf, ohne dass sie sich unterschieden. In einer bei uns gegenwärtig durchgeführten Studie [Wilhelm et al., 2000c] untersuchen wir bei Patienten mit Panikstörung detailliert die Veränderungen der Atmung im Verlauf eines intensiven Atemtraining-Programms, das durch $\mathrm{pCO}_{2}$-Feedback-Übungen zuhause begleitet wird. Hierbei werden sowohl im ambulanten Kontext als auch im Labor eine Reihe von Atemparametern erhoben, z.B. Baseline-PetCO 2 , Erholungsgeschwindigkeit des $\mathrm{PetCO}_{2}$ nach willkürlicher HV, und Variabilität des Atemzugvolumens. Erste Ergebnisse deuten auf die Effektivität des Interventionsverfahrens zur Normalisierung des $\mathrm{PetCO}_{2}$ hin.

Grossman et al. [1985] teilten 47 Patienten mit Angstsymptomen und HV-Syndrom in zwei Gruppen auf: Eine Gruppe nahm an einem Atemtraining mit durch auditives Feedback unterstütztem langsamen Atmen teil, die Vergleichsgruppe erhielt eine Placebobehandlung. In der Gruppe mit Atemtrai- ning reduzierten sich die Symptome stärker als in der Kontrollgruppe. Darüber hinaus, ging die Symptomverbesserung mit Veränderungen der Atmungsparameter einher. DeGuire et al. [1992] verglichen in ihrer Studie verschiedene Arten von Feedback-Therapie (Kapnographie, Atemgürtel, Therapeut ohne Geräteausstattung) mit einer Kontrollgruppe im Hinblick auf ihre symptomreduzierende Wirkung bei FHB. Diese war in allen Atemfeedback-Gruppen größer als in der Kontrollbedingung, ohne dass sich die Interventionsgruppen voneinander unterschieden. Die Erfolge konnten über mindestens 3 Jahre aufrechterhalten werden [DeGuire et al., 1996]. Die Symptomreduktion korrelierte eng mit der Abnahme der Atemfrequenz $(r=0,59, p<0,001)$ und mit dem $\mathrm{PetCO}_{2}$-Anstieg $(r=0,38, p<0,02)$. Dies deutet darauf hin, dass Veränderungen der Atmungsparameter mit einer Abnahme der FHBSymptome zusammenhängen und ihr möglicherweise zu Grunde liegen.

Atemtraining kann auch bei der Verbesserung von Spitzenleistungen sinnvoll sein. Einer der Autoren und ein Kollege [Bessel und Gevirtz, 1997] ordneten weibliche Turnerinnen mit Angst vor Leistungssituationen drei verschiedenen Gruppen zu: einer Kontrollgruppe, einer Gruppe mit kognitiver Therapie (mit Konzentration auf die Veränderung von Selbstinstruktionen vor der Leistungssituation) und eine Atemtrainingsgruppe (mit Kapnographie). Gemessen an den Bewertungen der Jury, verbesserte sich die Atemtrainingsgruppe am meisten (7,9 prä, 8,8 post), gefolgt von der Gruppe mit kognitiver Therapie. Die Kontrollgruppe verschlechterte sich geringfügig. Die Teilnehmer bewerteten die Atemtechniken als sehr hilfreiche Strategien im Umgang mit ihrer Leistungsangst.

\section{Klinische Gesichtspunkte}

Obwohl es noch an wissenschaftlichen Belegen für die Effektivität von Kapnographie-Feedback in Ergänzung zu reinem Atemtraining oder als Teil eines verhaltenstherapeutischen Programms mangelt, bewerten viele der in diesem Bereich arbeitenden Therapeuten dieses Verfahren als sehr wertvoll. Detaillierte Beschreibungen der spezifischen Vorgehensweisen finden sich bei Fried [1987]. In diesem Abschnitt versuchen wir, einige Prinzipien zusammenzufassen, die von erfahrenen Therapeuten in der Behandlung von Angststörungen, FHB oder chronischem Schmerz als nützlich eingeschätzt werden. Forschungsergebnisse zeigen, dass ein gewisser Anteil der Patienten mit diesen Störungen keine deutlichen Auffälligkeiten in Atmungsparametern aufweist. Für dies Untergruppen ist eine andere Vorgehensweise wahrscheinlich besser indiziert.

\section{Angststörungen}

Viele Therapeuten gehen davon aus, dass die Symptome der Panikstörung und zu einem gewissen Grad auch die von anderen Angststörungen mit einer Dysregulation der Atmung ein- 
hergehen. Verschiedene Therapieansätze, einschließlich der kognitiv-verhaltenstherapeutischen Standardbehandlungen für Panikstörung [z.B. Barlow et al., 1989], beinhalten Atemtrainingskomponenten. Einige Ansätze legen sogar ihren Schwerpunkt auf das Atemtraining. Ein vollständiges Atemtraining umfasst idealerweise folgende Schritte:

1) Analyse der Atmung während einer Baseline, bei Stressprovokation und während der Erholung mittels Kapnographie, eines Atemgürtels zur Erfassung des Atemmusters oder einfacher visueller Beobachtung der Atembewegungen,

2) Vermittlung von Hintergrundinformationen über den $\mathrm{Zu}$ sammenhang von Angst und physiologischen Veränderungen im Allgemeinen («Fight-or-flight»-Reaktionsmuster), und die physiologischen Zusammenhänge, die bei der Atmung eine Rolle spielen im Besonderen (z.B. Atemfrequenz, $\mathrm{pCO}_{2}$, Bohr-Effekt),

3) Vermittlung langsamer abdomineller Atmung mit einer Hand auf der Brust und einer auf Bauchhöhe,

4) wöchentlich stattfindende Sitzungen mit Feedback der Atemfrequenz, des $\mathrm{PetCO}_{2}$ und des Atemmusters und

5) Instruktionen zur Generalisierung der erlernten Strategien in Alltagssituationen.

Den Patienten wird verständlich gemacht, wie ihre Symptomatik durch ein Zusammenspiel von psychologischen Faktoren (Angst und die «Fight-or-flight»-Reaktion) und spezifischen physiologischen Prozessen (Aktivierung des sympathischen Nervensystems, Veränderung des $\mathrm{pCO}_{2}$ im Blut usw.) hervorgerufen werden kann. Ein Nebeneffekt dieser Vorgehensweise ist, dass sie entpathologisierend wirkt, besonders für Panikpatienten, die oft befürchten, eine schwere psychiatrische Erkrankung zu haben. Die mit der Therapie neu gewonnene Überzeugung, dass sie unter einer «psychophysiologischen» Störung leiden, trägt zur deutlichen Reduktion des mit der Störung verbundenen Stigmas bei. Schon in den ersten Sitzungen (Schritt 1 und 2) zeigen viele Patienten große Erleichterung, dass ihre Störung «real und nicht eingebildet» ist, da ihre erlebte Symptomatik durch Messungen objektiviert werden kann. Zusätzlich ist es vielen Patienten mit der Feedback-Methode möglich, zu verifizieren, dass die häuslichen Übungen wirklich «greifen», da sie Woche für Woche ihre Erfolge an der Normalisierung des $\mathrm{PetCO}_{2}$ beobachten können.

\section{Funktionelle Herzbeschwerden}

Das Behandlungsvorgehen bei FHB ist ähnlich zu dem oben beschriebenen Vorgehen. Der Schwerpunkt der Informationsvermittlung liegt hier auf dem Effekt der Atmung auf die Herzkranzgefässe und das restliche kardiovaskuläre System. Auch hier ist ein zentraler Schritt die Veränderung der Attribution des Patienten von einer «unerkannten Herzkrankheit» zu einem Krankheitsmodell, bei dem psychische Faktoren das physiologische System unter anderem durch veränderte Atmung beeinflussen. In eigenen Untersuchungen [DeGuire et al., 1996; DeGuire et al., 1992] konnten wir zeigen, dass sich die Patienten, die ihre Attributionen veränderten, am meisten verbesserten und diese Verbesserungen in den nächsten Jahren weiter ausbauten. Die Kapnographie ist möglicherweise nicht notwendig, scheint jedoch den Veränderungsprozess zu erleichtern.

\section{Chronischer Schmerz}

Wie oben beschrieben, werden chronische Schmerzen häufig von $\mathrm{HV}$ begleitet, welches sich auf lange Sicht ungünstig auf die Symptomatik auswirken kann. Demgemäß erscheint es logisch, dass ein Atemtraining bei der Behandlung dieser Beschwerden von Vorteil sein könnte. Derzeit liegen jedoch noch keine Daten zur Effektivitätsüberprüfung von Atemtrainings als einzige Intervention in der Behandlung von Patienten mit chronischen Schmerzen vor. Nach der klinischen Erfahrung von einem der Autoren (RG) kann das Atemtraining im Rahmen einer umfassenden Schmerzbewältigungstherapie eine wirkungsvolle Komponente darstellen. Dies entspricht auch den Annahmen der meisten körperorientierten Schmerztherapien [z.B. Clifton-Smith, 1998]. Auch hier können Kapnographie-Verlaufskurven zur Verdeutlichung der physiologischen Grundlage der Beschwerden genutzt werden. Hubbard, Gevirtz und Kollegen konnten zeigen, dass bei der Aufrechterhaltung von chronischen Muskelschmerzen eher eine sympathikotone Verbindung zu Muskelspindeln als zu den Muskelfasern bedeutsam ist [Banks et al., 1988; Gerwin et al., 1997; Gevirtz et al., 1996; Hubbard, 1996; Hubbard und Berkoff, 1993; McNulty et al., 1994]. Entspannung, die durch langsame abdominelle Atmung herbeigeführt wird, hat möglicherweise einen günstigen Effekt auf diese chronisch hyperaktive Sympathikusaktivität.

\section{Zusammenfassung und abschließende Bemerkungen}

Wir haben einige Grundlageninformationen zur Physiologie und Psychophysiologie der Atmung dargestellt. Bei einer Reihe von Beschwerden, bei denen psychophysiologische Mechanismen angenommen werden, scheint dieses einzigartige und komplexe physiologische System als Bindeglied zwischen Leib und Seele eine Rolle zu spielen. Die Befunde aus drei Bereichen - bei Panikstörung, FHB und chronischem Schmerz - unterstützen die Annahme, dass Prozesse, die mit der Atmung zusammenhängen, entweder als ätiologische Faktoren, Mediatoren oder als Marker fungieren. Technische Innovationen ermöglichen eine Messung des $\mathrm{PetCO}_{2}$ mit kleinen tragbaren Geräten im natürlichen Umfeld des Patienten, was für die Behandlung und Erforschung dieser Störungen neue Wege geöffnet hat.

Weiter haben wir therapeutische Vorgehensweisen beschrieben, die auf der angenommenen Mediatorenrolle der Atmung bei bestimmten Symptomen basieren. Dieser Bereich steckt noch in seinen Anfängen. Um die Gültigkeit eines stringenten Mediationsmodells nachzuweisen, müsste in Längsschnitt-Stu- 
dien herausgefunden werden, auf welche Weise eine Atmungsanomalie mit einer Störung kovariiert [Kraemer et al., 1997]. Mit solch einem Design könnte auch unterschieden werden, ob Atmungsanomalien Risikofaktoren für die Entwicklung der Beschwerden darstellen oder eher als Begleitphänomene anzusehen sind. Die oben aufgeführten klinischen Studien und Beobachtungen legen nahe, dass Therapien, die sich auf die Atmung konzentrieren, wirksam sind. Jedoch liegt ohne physiologische Messung kein Beweis vor, dass die Verbesserungen tatsächlich auf Atmungsvorgänge zurückzuführen sind. Nur sehr wenige Studien haben solche Messungen durchgeführt. Zusätzlich muss gezeigt werden, dass das Atemtraining einem vergleichbar glaubwürdigen Therapieverfahren überlegen ist. Ohne diesen Nachweis kann nicht ausgeschlossen werden, dass sogar der stärkste Therapieeffekt auf unspezifischen Faktoren beruht, d.h. einen Placeboeffekt darstellt. Doch auch der unspezifische Therapieeffekt kann von großem Interesse sein, wenn er mit ähnlich großen und anhaltenden Verbesserungen einhergeht wie die gängigen Standardtherapien. Es könnte von theoretischem und praktischem Nutzen sein, wenn die Therapie leichter zu verbreiten oder kosteneffektiver ist und weniger Nebenwirkungen als die Vergleichstherapien hat. Das Atemtraining vereinigt in sich viele dieser Eigenschaften, denn psychopharmakologische Behandlung geht mit kontinuierlichen Kosten und Nebenwirkungen einher, während kognitiv-behaviorale Therapieprogramme oft komplex aufgebaut sind und es derzeit noch an angemessen ausgebildeten Verhaltenstherapeuten mangelt. In jedem Fall erscheint bei den existierenden Behandlungen ein verstärkter Fokus auf die Atmung angebracht.

Unseres Erachtens ist zur gegenwärtigen Zeit das Verständnis über die komplexen Zusammenhänge von psychischen und körperlichen Faktoren bei vielen klinischen Syndromen noch nicht weit genug ausgereift, um einer Reihe von Patientengruppen auf die bestmögliche Weise zu helfen. Mit der Verbesserung der ätiologischen Modelle - und möglicherweise der Identifikation von Subtypen, bei denen Atmungsanomalien und anderen physiologischen Veränderungen eine besondere Rolle zukommt - werden auch die therapeutischen Möglichkeiten verbessert. Die angewandte Psychophysiologie sollte besonders nützlich sein, diese Behandlungen in medizinischen Settings theoretisch und praktisch akzeptabel $\mathrm{zu}$ machen, und auf diese Weise dazu beitragen, das Stigma zu überwinden, das häufig mit psychischen und psychosomatischen Erkrankungen einhergeht.

\section{Dank}

Mit Unterstützung des Department of Veterans Affairs und NIH Grant MH56094.

\section{References}

Abelson JL, Nesse RM, Weg JG, Curtis GC: Respiratory psychophysiology and anxiety: Cognitive intervention in the doxapram model of panic. Psychosom Med 1996a;58:302-313. Abelson JL, Weg JG, Nesse RM, Curtis GC: Persistent respiratory irregularity in patients with panic disorder. Biol Psychiatry 1996b;39: 521-522.

Aljadeff G, Molho M, Katz I, Benzaray S, Yemini Z, Shiner RJ: Pattern of lung volumes in patients with sighing breathing. Thorax 1993;48: 809-811.

Alpers GW, Wilhelm FH, Roth WT: Psychophysiological measures can index change in behavior therapy. Society for Psychophysiological Research, 40th Annual Meeting, San Diego, CA, USA, 2000.

American Psychiatric Association. Diagnostic and Statistical Manual of Mental Disorders, 4th ed. Washington, DC, American Psychiatric Association, 1994.

Aronson TA, Logue CM: Phenomenology of panic attacks: A descriptive study of panic disorder patients' self-reports. J Clin Psychiatry 1988;49:8-13.
Banks S, Jacobs D, Gevirtz R, Hubbard D: Effects of autogenic relaxation training on electromyographic activity in active myofascial trigger points. J Musculoskelet Pain 1998;64:23-32. Barlow DH, Craske MG, Cerny JA, Klosko JS: Behavioral treatment of panic disorder. Behav Ther 1989; 20:261-282.

Bass C: Management of patients with hyperventilation-related disorders; in Timmons BH, Ley R (ed): Behavioral and Psychological Approaches to Breathing Disorders. New York, Plenum Press, 1994, pp 149-156.

Bass C, Chambers J, Kiff P, Cooper D, Gardner $\mathrm{W}$ : Panic anxiety and hyperventilation in patients with chest pain: A controlled study. Q J Med 1988;69:949 -959.

Bessel J, Gevirtz R. (1997). Effects of breathing retraining versus cognitive techniques on cognitive and somatic components of state anxiety and on performence of female gymnasts. International Society for the Advancement of Respiratory Psychophysiology, Cape Cod, MA, USA, 1997.

Biber B, Alkin T: Panic disorder subtypes: Differential responses to $\mathrm{CO}_{2}$ challenge. Am J Psychiatry 1999;156: 739-744.
Briggs AC, Stretch DD, Brandon S: Subtyping of panic disorder by symptom profile. Br J Psychiatry 1993;163: 201-209.

Bystritsky A, Craske M, Maidenberg E, Vapnik T, Shapiro D: Autonomic reactivity of panic patients during a $\mathrm{CO}_{2}$ inhalation procedure. Depress Anxiety 2000;11: 15-26.

Clifton-Smith T: Breathing Works. Auckland, Penguin Press, 1998.

Conway AV: Breathing and feeling; in Timmons $\mathrm{BH}$, Ley R (ed): Behavioral and Psychological Approaches to Breathing Disorders. New York, Plenum Press, 1994, pp 243-252.

Creager B, Gevirtz R: The treatment of panic: A comparison study of breathing retraining and cognitive therapy; in Gevirtz R (ed): $\mathbf{\square}$. San Diego, $\mathbf{\square}, 2000, \mathrm{pp} \mathbf{\square}$

de Ruiter C, Ryken H, Garssen B, Kraaimaat F: Breathing retraining, exposure and a combination of both, in the treatment of panic disorder with agoraphobia. Behav Res Ther 1989;27: 647-655.

DeGuire S, Gevirtz R, Hawkinson D, Dixon K: Breathing retraining: A three-year follow-up study of treatment for hyperventilation syndrome and associated functional cardiac symptoms. Biofeedback Self Regul 1996;21:191-198. 
DeGuire S, Gevirtz R, Kawahara Y, Maguire $\mathrm{W}$ : Hyperventilation syndrome and the assessment of treatment for functional cardiac symptoms. Am J Cardiol 1992;70:673-677.

Ehlers A, Breuer P: How good are patients with panic disorder at perceiving their heartbeats? Biol Psychol 1996;42:165-182.

Finesinger JE: The spirogram in certain psychiatric disorders. Am J Psychiatry 1943;100: 159-169.

Fried R: The Hyperventilation Syndrome: Research and Clinical Treatment. Baltimore, John Hopkins University Press, 1987.

Fujii H, Yasue H, Okumura K, Matsuyama K, Morikami Y, Miyagi H, Ogawa H: Hyperventilation-induced simultaneous multivessel coronary spasm in patients with variant angina: An echocardiographic and arteriographic study. J Am Coll Cardiol 1988;12:1184-1192.

Gardner WN: Diagnosis and organic causes of symptomatic hyperventilation; in Timmons BH, Ley R (ed): Behavioral and Psychological Approaches to Breathing Disorders. New York, Plenum Press, 1994, pp 99-112.

Garssen B, Buikhuisen M, Hornsveld H, Klaver C, van Doornen L: Ambulatory measurement of transcutaneous $\mathrm{pCO}_{2}$. J Psychophysiol 1994; 8:231-240.

Garssen B, Buikhuisen M, van Dyck R: Hyperventilation and panic attacks. Am J Psychiatry 1996;153:513-518.

George DT, Nutt DJ, Walker WV, Porges SW, Adinoff B, Linnoila M: Lactate and hyperventilation substantially attenuate vagal tone in normal volunteers. A possible mechanism of panic provocation? Arch Gen Psychiatry 1989;46: 153-156.

Gerwin RD, Shannon S, Hong CZ, Hubbard D, Gevirtz R: Interrater reliability in myofascial trigger point examination. Pain 1997;69:65-73.

Gevirtz R, Hubbard, D, Harpin E: Psychophysiologic treatment of chronic low back pain. Prof Psychol Res Pract 1996;27:561-566.

Gilbert C: Hyperventilation and the body. Accid Emerg Nurs 1999;7:130-140.

Glynn C, Lloyd J, Folkhard S: Ventilatory response to intractable pain. Pain 1981;11:201-211. Gorman JM, Askanazi J, Liebowitz MR, Fyer A, Stein J, Kinney JM, Klein DF: Response to hyperventilation in a group of patients with panic disorder. Am J Psychiatry 1984;41: 857861.

Gorman JM, Fyer MR, Goetz R, Askanazi J, Liebowitz MR, Fyer AJ: Ventilatory physiology of patients with panic disorder. Arch Gen Psychiatry 1988a;45: 31-39.

Gorman JM, Goetz RR, Uy J, Ross D, Martinez J, Fyer AJ, Liebowitz MR, Klein DF: Hyperventilation occurs during lactate-induced panic. J Anxiety Disord 1988b; 2:193-202.

Gorman JM, Papp LA, Coplan JD, Martinez JM, Lennon S, Goetz RR, Ross D, Klein DF: Anxiogenic effects of $\mathrm{CO}_{2}$ and hyperventilation in patients with panic disorder. Am J Psychiatry 1994;151:547-553.
Grossman P, de Swart JC, Defares PB: A controlled study of a breathing therapy for treatment of hyperventilation syndrome. J Psychosom Res 1985;29:49-58.

Guyton A: Textbook of Medical Physiology, 9th ed. Philadelphia, Saunders, 1995.

Hegel MT, Ferguson RJ: Psychophysiological assessment of respiratory function in panic disorder: Evidence for a hyperventilation subtype. Psychosom Med 1997;59:224-230.

Hibbert G, Pilsbury D: Hyperventilation - is it a cause of panic attacks? Br J Psychiatry 1989; 155:805-809.

Hibbert GA, Pilsbury D: Hyperventilation in panic attacks. Ambulant monitoring of transcutaneous carbon dioxide. Br J Psychiatry 1988; 153:76-80.

Holt PD, Andrews G: Hyperventilation and anxiety in panic disorder, social phobia, GAD, and normal controls. Behav Res Ther 1998;27: 453-460.

Hornsveld H, Garssen B: Hyperventilation syndrome: An elegant but scientifically untenable concept. Neth J Med 1997;50:13-20.

Hubbard DR, Berkoff GM: Myofascial trigger points show spontaneous needle EMG activity. Spine 1993;18: 1803-1807.

Hubbard D: Chronic and Recurrent Muscle Pain: Pathophysiology and treatment, a review of pharmocologic studies. J Musculoskelet Pain 1996;4:123-143.

Ide Y, Hanaoka K, Tagami M, Nagase M, Numata $\mathrm{K}$, Yamamura H: The effects of hyperventilation upon the spinal pain modulating system (second report). Masui 1994a;43:294-298.

Ide Y, Hanaoka K, Tagami M, Nagase M, Numata $\mathrm{K}$, Yamamura $\mathrm{H}$ : Effects of hyperventilation upon the spinal pain modulating system (third report). Masui 1994b;43:1461-1466.

Kennealy JA, McLennan JE, Loudon RG, McLaurin RL: Hyperventilation-induced cerebral hypoxia. Am Rev Respir Dis 1980;122: 407-412.

Kerr WJ, Dalton JW, Gliebe PA: Some physical phenomena associated with the anxiety states and their relation to hyperventilation. Ann Intern Med 1937;11: 961-992.

Klein DF: Panic and phobic anxiety: Phenotypes, endophenotypes, and genotypes (editorial; comment). Am J Psychiatry 1998;155: 11471149.

Kraemer H, Kazdin A, Offord D, Kessler R, Jensen P, Kupfer D: Coming to terms with the terms of risk. Arch Gen Psychiatry 1997;54: 337-343.

Landis B, Romano PM: A scoring system for capnogram biofeedback: Preliminary findings. Appl Psychophysiol Biofeedback 1998;23: 75-91.

Ley R: Blood, breath, and fears: A hyperventilation theory of panic attacks and agoraphobia. Clin Psychol Rev 1985;5:271-285.

Ley R: The efficacy of breathing retraining and the centrality of hyperventilation in panic disorder: A reinterpretation of experimental findings. Behav Res Ther 1991;29:301-304.
Lum LC: Hyperventilation syndromes in medicine and psychiatry: A review. J R Soc Med 1987;80:229-231.

Maddock R, Carter C, Tavano-Hall L, Amsterdam E: Hypocapnia associated with cardiac stress scintigraphy in chest pain patients with panic disorder. Psychosom Med 1998;60:52-55. Maddock RJ, Carter CS: Hyperventilation-induced panic attacks in panic disorder with agoraphobia. Biol Psychiatry 1991;29:843-854.

Margraf J, Taylor CB, Ehlers A, Roth WT, Agras WS: Panic attacks in the natural environment. J Nerv Ment Dis 1987;175:558-565.

Martinez JM, Papp LA, Coplan JD, Anderson DE, Mueller CM, Klein DF, Gorman JM: Ambulatory monitoring of respiration in anxiety. Anxiety 1996;2: 296-302.

McNally RJ, Hornig CD, Donnell CD: Clinical vs. nonclinical panic: A test of the suffocation false alarm theory. Behav Ther Res 1995; 33:127-131.

McNulty WH, Gevirtz RN, Hubbard DR, Berkoff GM: Needle electromyographic evaluation of trigger point response to a psychological stressor. Psychophysiology 1994;31:313-316.

Munjack DJ, Brown RA, McDowell DE: Existence of hyperventilation in panic disorder with and without agoraphobia, GAD, and normals: Implications for a cognitive theory of panic. J Anxiety Disord 1993;7:37-48.

Nishino T, Shimoyama N, Ide T, Isono S: Experimental pain augments experimental dyspnea, but not vice versa in human volunteers. Anesthesiology 1999;91: 1633-1638.

Papp LA, Martinez JM, Klein DF, Coplan JD, Norman RG, Cole R, de Jesus MJ, Ross D Goetz R, Gorman JM: Respiratory psychophysiology of panic disorder: Three respiratory challenges in 98 subjects. Am J Psychiatry 1997; 154:1557-1565.

Pennebaker JW: Psychological factors influencing the reporting of physical symptoms; in Stone AA, Turkkan JS, Bachrach CA, Jobe JB, Kurtzman HS, Cain VS (eds): The Science of Self-Report: Implications for Research and Practice. Mahwah, Lawrence Erlbaum Associates, 2000, pp 299-315.

Pine DS, Coplan JD, Papp LA, Klein RG, Martinez JM, Kovalenko P, Tancer N, Moreau D, Dummit ES, Shaffer D, Klein DF, Gorman JM: Ventilatory physiology of children and adolescents with anxiety disorders. Arch Gen Psychiatry 1998;55:123-129.

Rapee R: Differential response to hyperventilation in panic disorder and generalized anxiety disorder. J Abnorm Psychol 1986;95:24-28.

Rapee RM, Brown TA, Antony MM, Barlow DH: Response to hyperventilation and inhalation of $5.5 \%$ carbon dioxide-enriched air across the DSM-III-R anxiety disorders. J Abnorm Psychol 1992;101:538-552. 
Salkovskis PM, Jones DR, Clark DM: Respiratory control in the treatment of panic attacks: Replication and extension with concurrent measurement of behaviour and $\mathrm{pCO}_{2}$. Br J Psychiatry 1986a;148:526-532.

Salkovskis PM, Jones DR, Clark DM: Respiratory control in the treatment of panic attacks: Replication and extension with concurrent measurement of behaviour and $\mathrm{pCO}_{2}$. Br J Psychiatry 1986b;148:526-532.

Schwartz GE, Goetz RR, Klein DF, Endicott J, Gorman JM: Tidal volume of respiration and 'sighing' as indicators of breathing irregularities in panic disorder patients. Anxiety 1996;2: 145-148.

Shioiri T, Someya T, Murashita J, Takahashi S: The symptom structure of panic disorder: A trial using factor and cluster analysis. Acta Psychiatr Scand 1996; 93:80-86.

Stein MB, Millar TW, Larsen DK, Kryger MH: Irregular breathing patterns during sleep in patients with panic disorder. Am J Psychiatry 1995;152:1168-1173.

Timmons BH, Ley R: Behavioral and Psychological Approaches to Breathing Disorders. New York, Plenum Press, 1994
Tobin MJ, Chadha TS, Jenouri G, Birch JS, Gazeroglu HB, Sackner MA: Breathing patterns. 2 diseased subjects. Chest 1983;84:286-294. van den Hout MA, Hoekstra R, Arntz A, Chris tiaanse M, Ranschaert W, Schouten E: Hyperventilation is not diagnostically specific to panic patients. Psychosom Med 1992;53:182-191.

Wheatley CE: Hyperventilation syndrome: A frequent cause of chest pain. Chest 1975;68: 195-199.

Wilhelm FH, Roth WT: Taking the laboratory to the skies: Ambulatory assessment of self-report, autonomic, and respiratory responses in flying phobia. Psychophysiology 1998;35:596606.

Wilhelm FH, Trabert W, Roth WT: Physiological instability in panic disorder and generalized anxiety disorder. Biol Psychiatry 2000a;(in press).

Wilhelm FH, Trabert W, Roth WT: Characteristics of sighing in panic disorder. Biol Psychiatry 2000b; (in press).
Wilhelm FH, Meuret AE, Roth WT: A new treatment for panic disorder: $\mathrm{pCO}_{2}$ and respiratory rate feedback assisted breathing training at home. 7th Annual Meeting of the International Society for the Advancement of Respiratory Psychophysiology, San Diego, USA, 2000c.

Woods SW, Charney DS, Loke J, Goodman WK, Redmond DE, Heninger GR: Carbon dioxide sensitivity in panic anxiety. Ventilatory and anxiogenic response to carbon dioxide in healthy subjects and patients with panic anxiety before and after alprazolam treatment. Arch Gen Psychiatry 1986;43:900-909.

Yasue H, Ogawa H, Okumura K: Coronary artery spasm in the genesis of myocardial ischemia. Am J Cardiol 1989;63:29E-32E.

Yu P, Yim B, Stanfield A: Hyperventilation syndrome: Changes in the electrocardiogram, blood gases, and electrolytes during voluntary hyperventilation: Possible mechanisms and clinical implications. Arch Intern Med 1959; 103: 902-913. 